\title{
On steepness of 3-jet non-degenerate functions
}

\author{
L. Chierchia ${ }^{1}$ (D) M. A. Faraggiana ${ }^{2} \cdot$ M. Guzzo ${ }^{3}$
}

Received: 8 August 2018 / Accepted: 13 April 2019 / Published online: 29 April 2019

(c) Fondazione Annali di Matematica Pura ed Applicata and Springer-Verlag GmbH Germany, part of Springer Nature 2019

\begin{abstract}
We consider geometric properties of 3-jet non-degenerate functions in connection with Nekhoroshev theory. In particular, after showing that 3-jet non-degenerate functions are "almost quasi-convex", we prove that they are steep and compute explicitly the steepness indices (which do not exceed 2) and the steepness coefficients.
\end{abstract}

Keywords Steepness · Steep functions · 3-Jet non-degeneracy $\cdot$ Nekhoroshev's theorem · Hamiltonian systems $\cdot$ Steepness indices $\cdot$ Exponential stability

Mathematics Subject Classification 34D20 $\cdot 37 \mathrm{~J} 40 \cdot 70 \mathrm{H} 08$

\section{Contents}

1 Introduction . . . . . . . . . . . . . . . . . . . . . . . 2152

2 Main result . . . . . . . . . . . . . . . . . . . . . . . . . 2155

3 Two lemmas . . . . . . . . . . . . . . . . . . . . . . . . . . . . . . 2159

4 Proof of the Theorem . . . . . . . . . . . . . . . . . . . . . 2161

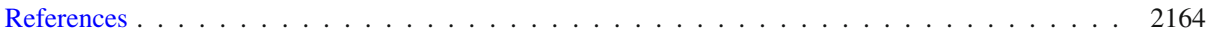

This research was partially supported by the Italian MIUR Grant 2015KB9WPT_008 "Variational methods, with applications to problems in mathematical physics and geometry".

$\bowtie$ L. Chierchia

luigi@mat.uniroma3.it

M. A. Faraggiana

martha.faraggiana@ubs.com

M. Guzzo

guzzo@math.unipd.it

1 Dipartimento di Matematica e Fisica, Università degli Studi Roma Tre, Largo San L. Murialdo 1, 00146 Rome, Italy

2 UBS - Zürich, Badenerstrasse 678, Altstetten, 8048 Zurich, Switzerland

3 Dipartimento di Matematica "Tullio Levi-Civita", Università degli Studi di Padova, Via Trieste 63, 35121 Padua, Italy 


\section{Introduction}

In 1977-1979, N.N. Nekhoroshev published a fundamental theorem [17-20] about the "exponential stability" of nearly integrable, real-analytic Hamiltonian systems with Hamiltonian given, in standard action-angle coordinates, by

$$
H(I, \varphi)=h(I)+\varepsilon f(I, \varphi), \quad(I, \varphi) \in U \times \mathbb{T}^{n},
$$

where $U \subseteq \mathbb{R}^{n}$ is an open region, $\mathbb{T}^{n}=\mathbb{R}^{n} /(2 \pi \mathbb{Z})^{n}$ is the standard flat $n$-dimensional torus and $\varepsilon$ is a small parameter. The integrable limit $h(I)$ is assumed to satisfy a geometric condition, called by Nekhoroshev "steepness", which can be formulated as follows (compare, also, Definition 2, § 2).

A function $f \in C^{1}(U)$, with $U$ a bounded region (i.e. open, bounded and connected set) of $\mathbb{R}^{n}$, is said to be steep in $U$ with steepness indices $\delta_{1}, \ldots, \delta_{n-1} \geq 1$ and (strictly positive) steepness coefficients $C_{1}, \ldots, C_{n-1}, \xi_{1}, \ldots, \xi_{n-1}$, if its gradient $h^{\prime}(I)$ satisfies the following estimates: $\inf _{I \in U}\left\|h^{\prime}(I)\right\|>0$ and, for any $I \in U$, for any $k$-dimensional linear subspace $V^{k} \subseteq \mathbb{R}^{n}$ orthogonal to $h^{\prime}(I)$ with $1 \leq k \leq n-1$, one has ${ }^{1}$

$$
\max _{0 \leq \eta \leq \xi} \min _{u \in V^{k}:\|u\|=\eta}\left\|P_{V^{k}} h^{\prime}(I+u)\right\| \geq C_{k} \xi^{\delta_{k}} \quad \forall \xi \in\left(0, \xi_{k}\right],
$$

where $P_{V^{k}}$ denotes the orthogonal projection over $V^{k}$.

Nekhoroshev's original exponential stability statement is, then, the following:

Let $H$ in (1) be real-analytic with $h$ steep. Then, there exist positive constants $a, b$ and $\varepsilon_{0}$ such that for any $0 \leq \varepsilon<\varepsilon_{0}$ the solution $\left(I_{t}, \varphi_{t}\right)$ of the (standard) Hamilton equations for $H(I, \varphi)$ with initial data $\left(I_{0}, \varphi_{0}\right)$ satisfies

$$
\left|I_{t}-I_{0}\right| \leq \varepsilon^{b}
$$

for any time t satisfying

$$
|t| \leq \frac{1}{\varepsilon} \exp \left(\frac{1}{\varepsilon^{a}}\right) .
$$

The values of the parameters $a, b, \epsilon_{0}$ in the original statements of [17-20], as well as in the recent improvement [6,7], depend on the steepness indices and coefficients. Precisely $a, b$ depend only on the values of the steepness indices and the number of the degrees of freedom, while $\epsilon_{0}$ depends also on the values of the steepness coefficients. In [7], the explicit dependence of $a, b, \epsilon_{0}$ on the steepness indices and parameters, as well as on the parameters depending on the perturbation $f$, is given, and the estimate of the stability exponent:

$$
a=\frac{1}{2 n \delta_{1} \cdot \delta_{n-2}}
$$

has been conjectured to be optimal.

Nekhoroshev proved in $[16,19,20]$ that steepness is a generic property of $C^{\infty}$ functions. Later, Niederman [21] proved that for real-analytic $h$, steepness is equivalent to require that $h$ has no critical points and that its restriction to any affine subspace of dimension $1 \leq k \leq n-1$ admits only isolated critical points. However, neither from Nekhoroshev's genericity techniques nor from Niederman's theorem there follow directly explicit conditions to determine whether a given function is steep or not. Indeed, very little is known about the evaluation of steepness parameters (index and coefficients) for general classes of functions,

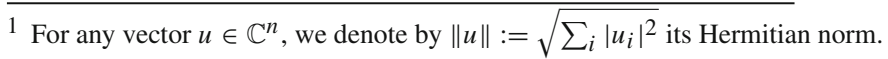


evaluation which is necessary in order to give explicit exponential estimates for perturbations of a specific steep Hamiltonian.

Essentially, the only general class of steep functions, which is well understood, is that of "quasi-convex" functions. Quasi-convexity is the simplest instance of steepness, and the quasi-convex case has been used for decades to improve the theoretical stability bounds of Nekhoroshev's theorem, especially the stability exponent $a$. In the quasi-convex case, the proof of the theorem has been significantly simplified (compare [2,3,5]), and furthermore, the stability exponent has been improved up to $a=(2 n)^{-1}$ (compare [11,12,24]; see, also, [4] for exponents which are intermediate between $a=(2 n)^{-1}$ and $\left.a=(2(n-1))^{-1}\right)$. Such exponents in the convex case have been proved to be nearly optimal [29].

Beyond the quasi-convex case, Nekhoroshev provided other sufficient conditions to recognize if a given $C^{k}$ function is steep in a neighbourhood of a point $I$. Such conditions are formulated in terms of the jet of partial derivatives of $h$ (compare [14-20]).

From this point of view, quasi-convex functions are identified as 2-jet non-degenerate functions. Precisely, in $[17,18]$, it is proved that if $\nabla h(I) \neq 0$, and the jet of order 2 of the function $h$ at $I$ is non-degenerate, i.e. if the system:

$$
\left\{\begin{array}{l}
\sum_{i=1}^{n} \frac{\partial h}{\partial I_{i}}(I) u_{i}=0 \\
\sum_{i, j=1}^{n} \frac{\partial^{2} h}{\partial I_{i} \partial I_{j}}(I) u_{i} u_{j}=0
\end{array}\right.
$$

has a unique solution $u=(0, \ldots, 0)$ in $\mathbb{R}^{n}$, then $h$ is steep in a neighbourhood of $I$ with steepness indices $\delta_{1}=\cdots=\delta_{n}=1$; the steepness coefficients follow from standard convexity estimates, since the restriction to any linear space $V^{k}$ orthogonal to $\nabla h(I)$ of a quasi-convex function $h\left(\right.$ or $^{2}-h$ ) is convex (compare, also, Remark (v), Sect. 2).

Therefore, one is left with the problem of computing steepness parameters of functions whose 2 -jet is degenerate.

In $[17,18]$, Nekhoroshev pointed out also the steepness of functions $h$ such that $\nabla h(I) \neq 0$ and with jet of order 3 at $I$ is non-degenerate, meaning that the system

$$
\left\{\begin{array}{l}
\sum_{i=1}^{n} \frac{\partial h}{\partial I_{i}}(I) u_{i}=0 \\
\sum_{i, j=1}^{n} \frac{\partial^{2} h}{\partial I_{i} \partial I_{j}}(I) u_{i} u_{j}=0 \\
\sum_{i, j, k=1}^{n} \frac{\partial^{3} h}{\partial I_{i} \partial I_{j} \partial I_{k}}(I) u_{i} u_{j} u_{k}=0
\end{array}\right.
$$

has a unique solution $u=(0, \ldots, 0)$ in $\mathbb{R}^{n}$.

Actually, steepness of 3-jet non-degenerate functions is not proved in [17,18], but rather it is obtained as a consequence of a former result of Nekhoroshev $[16,19,20]$ about the steepness of functions whose jets of order $j$ are outside the closure of the set $P_{j}$ of jets which satisfy

2 Steepness is invariant under the change $h \rightarrow-h$; hence, convexity and concavity are "equivalent" in this context and one usually refers only to convexity for simplicity. 
certain algebraic conditions ${ }^{3}$. From such papers, it follows that the steepness indices of 3 -jet non-degenerate functions are bounded from above with functions depending on $n$, and no computations of the steepness coefficients are provided.

Nevertheless, the 3-jet condition (being the only explicit general steepness condition apart from quasi-convexity) has gained quite a relevance in Nekhoroshev theory. Indeed, it enlarged significantly the range of applications of Nekhoroshev's Theorem, especially to celestial mechanics ( [1,10,13,22,23,25,27], see also the review paper [9]). Furthermore, numerical studies revealed the difference of the asymptotic stability between convex functions and 3 -jet non-degenerate functions (compare $[8,28]$ ).

On the other hand, 2-jet and 3-jet non-degeneracies, presently, appear to be the only algebraic sufficient conditions for steepness which are formulated with equations independent on the number $n$ of the degrees of freedom. For example, there are functions whose 4-jet is non-degenerate, in the sense that the system

$$
\left\{\begin{array}{l}
\sum_{i=1}^{n} \frac{\partial h}{\partial I_{i}}(I) u_{i}=0 \\
\sum_{i, j=1}^{n} \frac{\partial^{2} h}{\partial I_{i} \partial I_{j}}(I) u_{i} u_{j}=0 \\
\sum_{i, j, k=1}^{n} \frac{\partial^{3} h}{\partial I_{i} \partial I_{j} \partial I_{k}}(I) u_{i} u_{j} u_{k}=0 \\
\sum_{i, j, k, \ell=1}^{n} \frac{\partial^{4} h}{\partial I_{i} \partial I_{j} \partial I_{k} \partial I_{\ell}}(I) u_{i} u_{j} u_{k} u_{\ell}=0
\end{array}\right.
$$

has only the trivial solution $u=(0, \ldots, 0)$, but are not steep (see Example 1, [26])). Algebraic conditions for the steepness of functions of $n=3$ and $n=4$ variables which are 3-jet degenerate have been formulated in [26]; no general conditions for the steepness of 3-jet degenerate functions formulated using the 4-jet are known up to now. In fact, the sufficient jet conditions provided by Nekhoroshev in [14-16,19,20] are formulated in terms of the closure $C_{j}$ of a set whose definition depends on the number $n$ of the degrees of freedom; explicit equations for $C_{j}(n)$, valid for arbitrary $n$, are not known.

In this paper, we investigate further 3 -jet non-degenerate functions in connection with their steepness properties.

A key property of such functions is a "spectral non-degeneracy" of their Hessian matrix. More precisely, if $h$ is 3-jet non-degenerate and $V^{k}$ is a linear subspace of $h^{\prime}(I)^{\perp}$, then the symmetric operator $P_{V^{k}} h^{\prime \prime}(I): V^{k} \rightarrow V^{k}$ is strictly definite apart, possibly, from one single direction: in other words, there may be at most one small (or vanishing) eigenvalue; the precise statement is the content of Lemma 1 in Sect. 3. In this sense, one might say that 3-jet non-degenerate functions are "almost quasi-convex".

This observation allows to concentrate the study of steepness on lines (one-dimensional vector spaces) in $h^{\prime}(I)^{\perp}$ : this quantitative analysis is the content of Lemma 2 of Sect. 3.

Putting together these two facts, one can finally prove (Sect. 4) the steepness of 3 -jet non-degenerate functions and compute explicitly the steepness indices, which do not exceed 2 , and the steepness coefficients.

\footnotetext{
3 We remark that the result does not follow by simply checking if the 3-jet of a 3-jet non-degenerate function is outside the closure of $P_{3}$ but, depending on the value of $n$, there is suitably large $j$ such that the jet of a 3-jet non-degenerate function is outside the closure of $P_{j}$.
} 


\section{Main result}

We start with some standard notation:

- (Tensors of derivatives) Given $G \subseteq \mathbb{R}^{n}$ open, $p \in \mathbb{N}$ and a $C^{p}$ function $h: G \rightarrow \mathbb{R}$, $D^{p} h(I)=h^{(p)}(I)$ denotes the symmetric $p$-tensor at $I \in G$ of the $p$-derivatives acting on $^{4}\left(u_{1}, \ldots, u_{p}\right) \in\left(\mathbb{R}^{n}\right)^{p}$ as

$$
D^{p} h(I)\left[u_{1}, \ldots, u_{p}\right]:=\sum_{1 \leq i_{1}, \ldots, i_{p} \leq n} \frac{\partial^{p} h(I)}{\partial I_{i_{1}} \cdots \partial I_{i_{p}}} u_{1 i_{1}} \cdots u_{p i_{p}} .
$$

In particular, for $p=1, h^{(1)}$ is (identified with) the gradient

$$
h^{\prime}:=\nabla h:=\left(\frac{\partial h}{\partial I_{1}}, \ldots, \frac{\partial h}{\partial I_{n}}\right)
$$

and, for $p=2, h^{(2)}$ is (identified with) the Hessian matrix

$$
h^{\prime \prime}:=\left(\frac{\partial^{2} h}{\partial I_{i} \partial I_{j}}\right)_{i, j=1, \ldots, n} .
$$

For $n \geq p \geq 2, D^{p} h(I)\left[u_{2}, \ldots, u_{p}\right]$ denotes the vector in $\mathbb{R}^{n}$ with $i$ th-component given by:

$$
e_{i} \cdot D^{p} h(I)\left[u_{2}, \ldots, u_{p}\right]=D^{p} h(I)\left[e_{i}, u_{2}, \ldots, u_{p}\right]
$$

where $\left\{e_{1}, \ldots, e_{n}\right\}$ is the standard orthonormal bases of $\mathbb{R}^{n}\left(e_{i j}=\delta_{i j}\right)$ and $u \cdot v=$ $\sum_{i=1}^{n} u_{i} v_{i}$ the standard inner product in $\mathbb{R}^{n}$.

Analogously, for $n \geq p \geq 3, D^{p} h(I)\left[u_{3}, \ldots, u_{p}\right]$ denotes the $(n \times n)$-matrix with entries given by:

$$
D^{p} h(I)\left[u_{3}, \ldots, u_{p}\right] e_{i} \cdot e_{j}=D^{p} h(I)\left[e_{i}, e_{j}, u_{2}, \ldots, u_{p}\right] ;
$$

and so on for higher-order tensors (which, however, we shall not need).

Finally, for $n \geq p \geq k$ we shall also let

$$
D^{p} h(I)[u]^{k}:=D^{p} h(I)[\underbrace{u, \ldots, u}_{k \text { times }}] .
$$

- (Norms) In $\mathbb{R}^{n},\|x\|=\sqrt{x \cdot x}=\sqrt{\sum_{i=1}^{n} x_{i}^{2}}$ denotes Euclidean norm.

The norm of tensors of derivatives is the standard "functional norm":

$$
\begin{aligned}
\left\|D^{p} h(I)\right\|:=\sup _{\substack{u_{j}:\left\|u_{j}\right\|=1 \\
j=1, \ldots, p}}\left|D^{p} h(I)\left[u_{1}, \ldots, u_{p}\right]\right| \\
\left\|D^{p} h\right\|_{D}:=\sup _{D}\left\|D^{p} h(I)\right\| .
\end{aligned}
$$

${ }^{4} u_{i}=\left(u_{i 1}, \ldots, u_{i n}\right)$. 
From Cauchy-Schwarz inequality, there follows that

$$
\left\|D^{p} h\right\|_{D} \leq \sqrt{\sum_{1 \leq i_{1}, \ldots, i_{p} \leq n} \sup _{I \in D}\left|\frac{\partial^{p} h(I)}{\partial I_{i_{1}} \cdots \partial I_{i_{p}}}\right|^{2}}=: M_{p}
$$

- (Projections) In what follows, $V^{k}$ will denote a $k$-dimensional linear proper subspace of $\mathbb{R}^{n}, 1 \leq k \leq n-1$, and $P_{V^{k}}: \mathbb{R}^{n} \rightarrow V^{k}$ the orthogonal projection on $V^{k}:$ if $\left\{\bar{e}_{1}, \ldots, \bar{e}_{k}\right\}$ is an orthonormal basis of $V^{k}$, then

$$
P_{V^{k}} v=\sum_{j=1}^{k}\left(v \cdot \bar{e}_{j}\right) \bar{e}_{j}, \quad\left\|P_{V^{k}} v\right\|^{2}=\sum_{j=1}^{k}\left|v \cdot \bar{e}_{j}\right|^{2} .
$$

Recall that projections $P$ are symmetric operators with $\|P\| \leq 1$ and such that $P^{2}=P$.

Below, linear spaces $V^{k}$ will be always subspaces of the orthogonal complement of $h^{\prime}(I)$,

$$
h^{\prime}(I)^{\perp}:=\left\{u \in \mathbb{R}^{n} \mid u \cdot h^{\prime}(I)=0\right\},
$$

with $I$ regular point for $h$ (i.e. $h^{\prime}(I) \neq 0$ ).

We, now, recall the general notion of "jet non-degeneracy":

Definition 1 Let $p \in \mathbb{N}$ and $G \subseteq \mathbb{R}^{n}$ be open. A $C^{p}$ function $h: G \rightarrow \mathbb{R}$ is said to be $p$-jet non-degenerate at $I \in G$ if

$$
D^{k} h(I)[u]^{k}=0, \quad \forall 1 \leq k \leq p \quad \Longrightarrow \quad u=0
$$

The function $h$ is said to be $p$-jet non-degenerate on $D \subseteq G$ if $h$ is $p$-jet non-degenerate at every $I \in D$.

Remarks (i) A 1 -jet non-degenerate function at $I$ is simply a function regular at $I$, i.e. such that $h^{\prime}(I) \neq 0$.

A 2-jet non-degenerate function with nonvanishing gradient is, by definition, a quasiconvex function (at $I$ ); in other words, a quasi-convex function is a function $h$ which is strictly convex (or concave) on $h^{\prime}(I)^{\perp}, I$ being a regular point for $h$.

(ii) From (6), it follows immediately that if $h$ is 2 -jet non-degenerate with nonvanishing gradient (quasi-convex) on a compact set $D \subseteq G$ then,

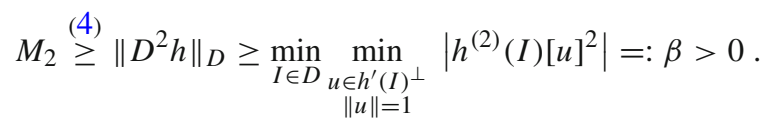

Analogously, if $h$ is 3-jet non-degenerate at $I$ with nonvanishing gradient, then

$$
M_{3} \stackrel{(4)}{\geq}\left\|D^{3} h\right\|_{D} \geq \min _{\substack{u \in h^{\prime}(I)^{\perp} \\\|u\|=1}} \max \left\{\left|h^{(2)}(I)[u]^{2}\right|,\left|h^{(3)}(I)[u]^{3}\right|\right\}:=\beta(I)>0,
$$


and, if $h$ is 3-jet non-degenerate on a compact set $D \subseteq G$, then ${ }^{5}$

$$
M_{3} \geq \min _{\substack{I \in D,\|u\|=1 \\ u \in h^{\prime}(I)^{\perp}}} \max \left\{\left|h^{(2)}(I)[u]^{2}\right|,\left|h^{(3)}(I)[u]^{3}\right|\right\}:=\beta>0,
$$

(obviously, $\beta(I) \geq \beta$ ).

(iii) For every $v \in \mathbb{R}^{n}$ and for every $u \in V^{k}$ with $\|u\|=1$, one has

$$
\left\|P_{V^{k}} v\right\| \geq\left|P_{V^{k}} v \cdot u\right|=\left|v \cdot P_{V^{k}} u\right|=|v \cdot u| .
$$

Applying these inequalities with $v=h^{\prime \prime}(I) u$, one sees that if $h$ is 2-jet non-degenerate on $D$ it follows that, $\forall I \in D, \forall V^{k} \subseteq h^{\prime}(I)^{\perp}$,

$$
\left\|P_{V^{k}} h^{\prime \prime}(I) u\right\| \geq \beta, \quad \forall u \in V^{k},\|u\|=1 .
$$

Analogously, applying (9) with $v=h^{(2)}(I) u$ and $v=h^{(3)}(I)[u]^{2}$ one sees that if $h$ is 3-jet non-degenerate on $D$ it follows that, $\forall I \in D, \forall V^{k} \subseteq h^{\prime}(I)^{\perp}$,

$$
\max \left\{\left\|P_{V^{k}} h^{\prime \prime}(I) u\right\|,\left\|P_{V^{k}} h^{(3)}(I)[u]^{2}\right\|\right\} \geq \beta, \quad \forall u \in V^{k},\|u\|=1 .
$$

Notice also that the eigenvalues of $P_{V^{k}} h^{\prime \prime}(I)$ have absolute value bounded by $M_{2}$ : indeed, if $P_{V^{k}} h^{\prime \prime}(I) \bar{e}=\lambda \bar{e}$ with $\|\bar{e}\|=1$, then

$$
|\lambda|=\|\lambda \bar{e}\|=\left\|P_{V^{k}} h^{\prime \prime}(I) \bar{e}\right\| \leq M_{2} .
$$

Let us now turn to the definition of steepness as originally given by N.N. Nekhoroshev:

Definition 2 (Nekhoroshev [17,18]) Let $n \geq 2$ be an integer and $G \subseteq \mathbb{R}^{n}$ an open set. A $C^{1}$ function $h: G \subseteq \mathbb{R}^{n} \rightarrow \mathbb{R}$ is said to be steep at a point $I \in G$ if $I$ is a regular point for $h$ (i.e. $h^{\prime}(I) \neq 0$ ) and, for each $1 \leq k \leq n-1$, there exist positive constants $C_{k}, \xi_{k}, \delta_{k}$ such that the inequality

$$
\max _{0 \leq \eta \leq \xi} \min _{\substack{u \in V^{k} \\\|u\|=1}}\left\|P_{V^{k}} h^{\prime}(I+\eta u)\right\| \geq C_{k} \xi^{\delta_{k}}, \forall 0<\xi \leq \xi_{k}
$$

holds for every linear subspace $V^{k} \subseteq h^{\prime}(I)^{\perp}$.

The numbers $C_{k}$ and $\xi_{k}$ are called steepness coefficients, while $\delta_{k}$ is called the steepness index of order $k$.

A $C^{1}$ function $h$ is said to be steep on $D \subseteq G$ if there exist positive constants $C_{k}, \xi_{k}, \delta_{k}$ such that, for every $I \in D, h$ is steep at $I$ with coefficients $C_{k}, \xi_{k}$ and indices $\delta_{k}$.

Let us make a few more remarks.

(iv) Definition 2 is well posed since the function (defined for $\eta$ small enough)

$$
\eta \rightarrow F_{V^{k}}(\eta):=\min _{\substack{u \in V^{k} \\\|u\|=1}}\left\|P_{V^{k}} h^{\prime}(I+\eta u)\right\|
$$

is upper semi-continuous, and hence, it achieves maximum on compact sets (note, however, that $\left.F_{V^{k}}(0)=0\right)$.

$\overline{5}$ Notice that, if $S^{n-1}:=\left\{u \in \mathbb{R}^{n} \mid\|u\|=1\right\}$, the function $F:(I, u) \in D \times S^{n-1} \rightarrow F(I, u):=$ $\max \left\{\left|h^{(2)}(I)[u]^{2}\right|,\left|h^{(3)}(I)[u]^{3}\right|\right\}$ is continuous on the compact set $\left\{(I, u) \in D \times S^{n-1} \mid h^{\prime}(I) \cdot u=0\right\}$ and therefore attains a minimum $\beta$ on such a set: such a minimum is strictly positive since when $h^{\prime}(I) \cdot u=0$, by (3), $F(I, u)>0$. 
(v) Quasi-convex functions are the steepest functions: they are steep with lowest possible indices, namely $\delta_{k}=1$ for all $k$.

Let us recall the elementary argument: by (10) and by Taylor's formula, for all $u \in V^{k} \cap G$ with $\|u\|=1, V^{k}$ linear subspace of $h^{\prime}(I)^{\perp}$, and for small enough $\xi>0$, one has:

$$
\begin{aligned}
\left\|P_{V^{k}} h^{\prime}(I+\xi u)\right\| & =\left\|P_{V^{k}} h^{\prime}(I)+\xi P_{V^{k}} D^{2} h(I) u+o(\xi)\right\| \\
& =\left\|\xi P_{V^{k}} h^{\prime \prime}(I) u+o(\xi)\right\| \\
& \geq \xi\left(\left\|P_{V^{k}} h^{\prime \prime}(I) u\right\|-o(1)\right) \stackrel{(10)}{\geq} \frac{\beta}{2} \xi,
\end{aligned}
$$

and steepness at $I$ follows with $\delta_{k}=1$ for all $k$ (and $C_{k}=\beta / 2$ ). The argument extends uniformly on compact sets.

Notice also that this proves a stronger property than steepness since it has been enough to consider only $\eta=\xi$ in (13) (rather than all $0<\eta \leq \xi$ ).

We are ready to formulate the main result:

Theorem Let $D$ be a compact subset of an open set $G \subseteq \mathbb{R}^{n}$ such that $B_{r}(I) \subseteq G$ for all $I \in D$. Let $h: G \rightarrow \mathbb{R}$ be a $C^{4}$ function and assume that $h^{\prime} \neq 0$ on $D$ and that $h$ is 3-jet non-degenerate on $D$. Let $\beta$ as in (8), $M_{p}$ as in (4) and define

$$
M:=\max \left\{M_{2}, M_{3}, M_{4}\right\}, \quad \gamma:=\frac{\beta}{M}, \quad \theta:=\frac{1}{8(3+2 \sqrt{2})}
$$

and, for $1 \leq k \leq n-1$,

$$
C_{k}=C:=\theta \beta, \quad \xi_{k}:=\min \left\{r, \frac{\theta}{2 k^{2}} \gamma^{3}\right\} .
$$

Then, $h$ is steep on $D$ with coefficients $C_{k}, \xi_{k}$ and indices $\delta_{k} \leq 2$.

Remarks (vi) From the definitions given it follows immediately that

$$
\gamma \leq 1, \quad \xi_{k} \leq \frac{1}{16(3+2 \sqrt{2})} .
$$

(vii) We shall first prove steepness for the third-order truncation of the Taylor expansion of $h$ and then extend it to the full function: this is not surprising, as the main hypothesis regards the 3-jet of $h$, which may be identified with the third-order Taylor polynomial of $h$. For this purpose, let us denote $\bar{h}$ the third-order Taylor polynomial of $h$ at $^{6} I \in D$ :

$$
\bar{h}\left(I^{\prime}\right):=\sum_{j=0}^{3} \frac{1}{j !} D^{j} h(I)\left[I^{\prime}-I\right]^{j}
$$

so that by Taylor's formula with integral remainder it is

$$
\begin{aligned}
& h^{\prime}(I+u)=\bar{h}^{\prime}(I+u)+R(u ; I), \\
& R(u ; I):=\frac{1}{2} \int_{0}^{1}(1-t)^{2} h^{(4)}(I+t u)[u]^{3} d t, \\
& \|R(u ; I)\| \leq \frac{M_{4}}{6}\|u\|^{3}, \quad \forall I \in D,\|u\| \leq r .
\end{aligned}
$$

$6 D^{0} h:=h$. 
We shall also define the truncated "Nekhoroshev's function", for given $I \in D$ and $V^{k} \subseteq h(I)^{\perp}$,

$$
\bar{F}_{V^{k}}(\eta):=\min _{\substack{u \in V^{k} \\\|u\|=1}}\left\|P_{V^{k}} \bar{h}^{\prime}(I+\eta u)\right\| .
$$

Thus, from definitions (14), (18) and (16), one has

$$
F_{V^{k}}(\eta) \geq \bar{F}_{V^{k}}(\eta)-\frac{M_{4}}{6} \eta^{3}
$$

(viii) Some of the steepness indices $\delta_{k}$ of 3 -jet non-degenerate functions can be equal to 1 ; this happens (trivially) for quasi-convex functions where $\delta_{k}=1$ for all $k$. Also, $\delta_{k}=1$ for some $k$ if the restriction of the Hessian matrix of $h$ to any $k$-dimensional linear space orthogonal to $h^{\prime}(I)$ is non-degenerate $[1,8]$.

\section{Two lemmas}

In this section, we show two properties of 3-jet non-degenerate functions: the first is a simple but crucial spectral non-degeneracy property, namely that the restriction of the Hessian of a 3-jet non-degenerate function on a linear space orthogonal to its gradient has at most one "small" eigenvalue: 3-jet non-degenerate functions are "almost quasi-convex".

The second property is the direct, explicit check of steepness of 3-jet non-degenerate functions on lines (one-dimensional linear subspaces).

These two properties together lead to a simple proof of steepness (given in Sect. 4).

Lemma 1 (Almost quasi-convexity) If $h$ is 3-jet non-degenerate at $I$, and $V^{k} \subseteq h^{\prime}(I)^{\perp}$ with $k \geq 2$, then the spectrum of $P_{V^{k}} h^{\prime \prime}(I): V^{k} \rightarrow V^{k}$ has at most one eigenvalue in absolute value strictly smaller than $\beta(I)$, where $\beta(I)$ is defined in (7).

Proof Assume, by contradiction, that the conclusion is false. Then, there is an orthonormal basis of eigenvectors $\left\{\bar{e}_{1}, \ldots, \bar{e}_{k}\right\} \subseteq V^{k}$ of $P_{V^{k}} h^{\prime \prime}(I)$ with corresponding eigenvalues $\lambda_{k}$ so that $\left|\lambda_{1}\right| \leq \cdots \leq\left|\lambda_{k}\right|$ and $\left|\lambda_{1}\right| \leq\left|\lambda_{2}\right|<\beta(I)$. For $t \in[0,2 \pi]$, consider the unitary vectors in $V^{k}$ given by $u_{t}:=(\cos t) \bar{e}_{1}+(\sin t) \bar{e}_{2}$. Then,

$$
\left|h^{\prime \prime}(I) u_{t} \cdot u_{t}\right|=\left|P_{V^{k}} h^{\prime \prime}(I) u_{t} \cdot u_{t}\right|=\left|\lambda_{1} \cos ^{2} t+\lambda_{2} \sin ^{2} t\right| \leq\left|\lambda_{2}\right|<\beta(I)
$$

but this implies, by 3 -jet non-degeneracy and the definition of $\beta(I)$, that $\left|h^{(3)}\left[u_{t}\right]^{3}\right| \geq \beta(I)$ for any $t$, and this is not possible since the real continuous function $t \in[0,2 \pi] \rightarrow h^{(3)}\left[u_{t}\right]^{3}$ changes $\operatorname{sign}^{7}$ and hence must have a zero.

Lemma 2 (Steepness on lines) Under the assumptions of the Theorem, let $\theta_{0}:=4 \theta$

$$
\kappa:=\theta_{0} \beta, \quad c:=\sqrt{2 \kappa / M} .
$$

For every $I \in D, u \in h^{\prime}(I)^{\perp}$ with $\|u\|=1$ and $0<\xi \leq \gamma$, there exists $\eta=\eta_{u, \xi}$ such that ${ }^{8}$

$$
c \xi \leq \eta_{u, \xi} \leq \xi
$$

and

$$
\bar{F}_{V_{u}^{1}}\left(\eta_{u, \xi}\right)=\min _{\sigma= \pm 1}\left|\bar{h}^{\prime}\left(I+\sigma \eta_{u, \xi} u\right) \cdot u\right| \geq \kappa \xi^{2}
$$

\footnotetext{
7 For example, $h^{(3)}\left[u_{0}\right]^{3}=-h^{(3)}\left[u_{\pi}\right]^{3}$.

8 Notice that $2 \kappa / M=\beta /(M(3+2 \sqrt{2}))<1$, so that $c<1$.
} 
where we have denoted by $V_{u}^{1}$ the 1-dimensional space generated by $u$ :

$$
V_{u}^{1}:=\{t u \mid t \in \mathbb{R}\} .
$$

Proof The equality in (22) follows from representation (5) with $k=1$ and observing that $\left\{u^{\prime} \in V_{u}^{1}:\left\|u^{\prime}\right\|=1\right\}=\{ \pm u\}$.

Since $\bar{h}$ is the third-order Taylor polynomial of $h$ at $I$, for $\sigma= \pm 1$, it is:

$$
\bar{F}_{V_{u}^{1}}(\eta)=\left|P_{V_{u}^{1}} \bar{h}(I+\sigma \eta u)\right|=\left|\sigma \eta P_{V_{u}^{1}} h^{\prime \prime}(I) u+\frac{\eta^{2}}{2} P_{V_{u}^{1}} h^{(3)}[u]^{2}\right|
$$

so that

$$
\bar{F}_{V_{u}^{1}}(\eta) \geq\left|a \eta-\frac{b}{2} \eta^{2}\right|
$$

having set $a:=\left\|P_{V_{u}^{1}} h^{\prime \prime}(I) u\right\|$ and $b:=\left\|P_{V_{u}^{1}} h^{(3)}[u]^{2}\right\|$. Note that, by (11), (4) and the definition of $M$, it is

$$
\beta \leq \max \{a, b\} \leq M
$$

We consider various cases.

$$
a \geq \beta
$$

Taking $\eta_{u, \xi}=\xi$ :

$$
\bar{F}_{V_{u}^{1}}\left(\eta_{u, \xi}\right) \stackrel{(23)}{\geq} a \xi-\frac{b}{2} \xi^{2} \stackrel{(a),(24)}{\geq} \beta \xi-\frac{M}{2} \xi^{2} \geq \frac{\beta}{2} \xi>\kappa \xi^{2}
$$

where in the last two inequalities we used, respectively,

$$
\xi \leq \gamma:=\beta / M, \quad \frac{\beta}{2 \kappa} \stackrel{(20)}{=}(3+2 \sqrt{2})>1 .
$$

Next case is:

$$
a=0
$$

In view of (24), this implies $b \geq \beta$. Then, taking $\eta_{u, \xi}=\xi$ :

$$
\bar{F}_{V_{u}^{1}}\left(\eta_{u, \xi}\right)=\frac{\xi^{2}}{2} b \geq \frac{\xi^{2}}{2} \beta \stackrel{(20)}{=}(3+2 \sqrt{2}) \kappa \xi^{2}>\kappa \xi^{2} .
$$

We are left with the case:

$$
0<a<\beta \text {. }
$$

Note that, again because of (24),

$$
0<a<\beta \stackrel{(24)}{\leq} b \leq M .
$$

Let $\alpha:=\sqrt{2 \kappa / b}$ and observe that

$$
c=\sqrt{\frac{2 \kappa}{M}} \leq \alpha \leq \sqrt{\frac{2 \kappa}{\beta}} \stackrel{(20)}{=} \frac{1}{\sqrt{1+\sqrt{2}}} .
$$


We then have three subcases:

$$
\alpha \xi>\frac{a}{b}
$$

In this case, we choose

$$
\eta_{u, \xi}:=\frac{a+\sqrt{a^{2}+2 \kappa b \xi^{2}}}{b}=\frac{a}{b}+\sqrt{\left(\frac{a}{b}\right)^{2}+\alpha^{2} \xi^{2}}
$$

so that

$$
\left|a \eta_{u, \xi}-\frac{b}{2} \eta_{u, \xi}^{2}\right|=b \eta_{u, \xi}\left|\frac{a}{b}-\frac{\eta_{u, \xi}}{2}\right|=\frac{b}{2} \alpha^{2} \xi^{2}=\kappa \xi^{2},
$$

showing, by (23), that (22) is satisfied. Furthermore, by the hypothesis $a / b<\alpha \xi^{2}$, (26) and the definition of $\kappa$ in (20), one finds

$$
c \xi \stackrel{(26)}{\leq} \alpha \xi \stackrel{(27)}{\leq} \eta_{u, \xi} \stackrel{\left(\mathrm{c}_{1}\right)}{<} \alpha \xi(1+\sqrt{2}) \stackrel{(26)}{\leq} \xi,
$$

proving (21).

Next, we consider the case

$$
\xi \geq \frac{a}{b} \geq \alpha \xi
$$

and choose $\eta_{u, \xi}:=a / b$. We then find

$$
\left|a \eta_{u, \xi}-\frac{b}{2} \eta_{u, \xi}^{2}\right|=\frac{b}{2}\left(\frac{a}{b}\right)^{2} \geq \frac{b}{2} \alpha^{2} \xi^{2}=\kappa \xi^{2},
$$

showing, again by (23), that (22) is satisfied. Inequalities (21) follow immediately by the hypothesis and the fact that $c \leq \alpha$.

The final case is

$$
\frac{a}{b}>\xi
$$

We choose again $\eta_{u, \xi}=\xi$, so that:

$$
\left|a \xi-\frac{b}{2} \xi^{2}\right| \geq b\left(\frac{a}{b} \xi-\frac{\xi^{2}}{2}\right)>\frac{b}{2} \xi^{2} \stackrel{(25)}{\geq} \frac{\beta}{2} \xi^{2} \stackrel{(26)}{>} \kappa \xi^{2} .
$$

\section{Proof of the Theorem}

First we prove steepness for the third-order polynomial truncation of $h$.

For $k=1$, steepness for the third-order polynomial truncation of $h$ follows from Lemma 2 . We therefore assume $2 \leq k \leq n-1$, fix $I \in D$, fix $V^{k}$ a linear space of dimension $k$ in $h(I)^{\perp}$ and let, as above, $\bar{h}$ denote the third-order Taylor polynomial of $h$ at $I$. Let $\left\{\bar{e}_{1}, \ldots, \bar{e}_{k}\right\} \subseteq V^{k}$ be an orthonormal basis of eigenvectors of $P_{V^{k}} h^{\prime \prime}(I)$ with corresponding eigenvalues $\lambda_{k}$ so that $\left|\lambda_{1}\right| \leq \cdots \leq\left|\lambda_{k}\right|$. Then, by Lemma 1 and (12) one has

$$
\beta \leq\left|\lambda_{j}\right|, \forall j \geq 2 ; \quad\left|\lambda_{j}\right| \leq M_{2} \leq M, \forall j .
$$

Fix $0<\xi \leq \xi_{k}$ and a unit vector $u \in V^{k}$ and define

$$
\bar{\eta}:=\eta_{\bar{e}_{1}, \xi}
$$


with $\eta_{\bar{e}_{1}, \xi}$ as in Lemma 2 . Recall that, by (21), it is

$$
c \xi \leq \bar{\eta} \leq \xi .
$$

We claim that

$$
\bar{F}_{V^{k}}(\bar{\eta})=\min _{\substack{u \in V^{k} \\\|u\|=1}}\left\|P_{V^{k}} \bar{h}^{\prime}(I+\bar{\eta} u)\right\| \geq \frac{\kappa}{2} \xi^{2}, \quad \forall 0<\xi \leq \xi_{k} .
$$

Estimate (31) says that steepness on $V^{k}$ can be controlled in terms of steepness along the line in $V^{k}$ corresponding to the "degenerate" eigenvector $\bar{e}_{1}$ of $P_{V^{k}} h^{\prime \prime}(I)$, where degeneracy means here that $\left|\lambda_{1}\right|$ may be smaller in absolute value than $\beta$ (and even vanish).

To prove the claim, we let

$$
u=\sum_{j=1}^{k} x_{j} \bar{e}_{j}, \quad \sum_{j=1}^{k} x_{j}^{2}=1,
$$

be the expansion of $u$ in the orthonormal basis $\left\{\bar{e}_{j}\right\}$ and fix

$$
v:=k \frac{M}{\beta}=\frac{k}{\gamma} \text {. }
$$

Notice that

$$
2 \leq k \leq v, \quad \xi_{k} \stackrel{(15)}{\leq} \frac{2 \theta}{3 k} \frac{1}{v}<\frac{1}{v} .
$$

We distinguish two cases: first, assume that:

$$
\sum_{j=2}^{k} x_{j}^{2} \geq v^{2} \bar{\eta}^{2}
$$

In this case, recalling (28), we have

$$
\sum_{j=2}^{k}\left|\lambda_{j}\right|\left|x_{j}\right| \geq \sqrt{\sum_{j=2}^{k}\left|\lambda_{j}\right|^{2}\left|x_{j}\right|^{2}} \geq \beta \sqrt{\sum_{j=2}^{k} x_{j}^{2}} \stackrel{\text { (A) }}{\geq} \beta \nu \bar{\eta} .
$$

Then, observe that, for all $1 \leq j \leq k$,

$$
\begin{aligned}
\left\|P_{V^{k}} \bar{h}^{\prime}(I+\bar{\eta} u)\right\| & \geq\left\|P_{V^{k}} \bar{h}^{\prime}(I+\bar{\eta} u) \cdot \bar{e}_{j}\right\| \geq \bar{\eta}\left\|P_{V^{k}} h^{(2)}(I) u \cdot \bar{e}_{j}\right\|-\frac{\bar{\eta}^{2}}{2} M \\
& =\bar{\eta}\left|\lambda_{j}\right|\left|x_{j}\right|-\frac{\bar{\eta}^{2}}{2} M,
\end{aligned}
$$

so that, summing over $2 \leq j \leq k$, one gets

$$
\begin{aligned}
\left\|P_{V^{k}} \bar{h}^{\prime}(I+\bar{\eta} u)\right\| & \geq \frac{\bar{\eta}}{k} \sum_{2=1}^{k}\left|\lambda_{j}\right|\left|x_{j}\right|-\frac{\bar{\eta}^{2}}{2} M \\
& \stackrel{(34)}{\geq} \bar{\eta}^{2}\left(\frac{\beta v}{k}-\frac{M}{2}\right) \stackrel{(32)}{=} \bar{\eta}^{2} \frac{M}{2} \\
& \stackrel{(30)}{\geq} c^{2} \frac{M}{2} \xi^{2}=\kappa \xi^{2}>\frac{\kappa}{2} \xi^{2},
\end{aligned}
$$

proving the claim (31) in case (A). 
Assume now that

$$
\sum_{j=2}^{k} x_{j}^{2}<v^{2} \bar{\eta}^{2}
$$

Notice that by (33) $\nu \bar{\eta} \leq \nu \xi_{k}<1$ so that $\sum_{j=2}^{k} x_{j}^{2}<1$ and, hence, $x_{1} \neq 0$.

If $\sum_{j=2}^{k} x_{j}^{2}=0$, i.e. $x_{1}= \pm 1$, the claim follows directly from Lemma 2 in view of the choice of $\bar{\eta}$ in (29).

Therefore, we assume $0<\left|x_{1}\right|<1$. Assumption (B) implies that $\left|x_{1}\right|$ is close to 1 :

$$
1-\left|x_{1}\right|<1-x_{1}^{2}=\sum_{j=2}^{k} x_{j}^{2} \stackrel{(\mathrm{B})}{<} v^{2} \bar{\eta}^{2} .
$$

Let $\sigma=\operatorname{sign}\left(x_{1}\right)$ so that

$$
x_{1}-\sigma=\sigma\left(\left|x_{1}\right|-1 \mid\right) .
$$

Then, recalling (29), by Lemma 2, one has

$$
\left|\bar{h}^{\prime}\left(I+\sigma \bar{\eta} \bar{e}_{1}\right) \cdot \bar{e}_{1}\right| \geq \kappa \xi^{2} .
$$

We want to approximate $\bar{h}^{\prime}(I+\bar{\eta} u) \cdot \bar{e}_{1}$ with $\bar{h}^{\prime}\left(I+\sigma \bar{\eta} \bar{e}_{1}\right) \cdot \bar{e}_{1}$. We do it in two steps.

First, expanding $u$ in the eigen-base $\left\{\bar{e}_{j}\right\}$ and cancelling out the equal terms, we find:

$$
\begin{aligned}
& \bar{h}^{\prime}(I+\bar{\eta} u) \cdot \bar{e}_{1}-\bar{h}^{\prime}\left(I+\bar{\eta} x_{1} \bar{e}_{1}\right) \cdot \bar{e}_{1} \\
& =\bar{\eta} h^{\prime \prime}(I) u \cdot \bar{e}_{1}-\bar{\eta} x_{1} h^{\prime \prime}(I) \bar{e}_{1} \cdot \bar{e}_{1}+\frac{\bar{\eta}^{2}}{2} h^{(3)}(I)\left[\bar{e}_{1}, u, u\right]-\frac{\bar{\eta}^{2}}{2} x_{1}^{2} h^{(3)}(I)\left[\bar{e}_{1}\right]^{3} \\
& =\frac{\bar{\eta}^{2}}{2} h^{(3)}(I)\left[\bar{e}_{1}, u, u\right]-\frac{\bar{\eta}^{2}}{2} x_{1}^{2} h^{(3)}(I)\left[\bar{e}_{1}\right]^{3} \\
& =\frac{\bar{\eta}^{2}}{2} \sum_{(i, j) \neq(1,1)} x_{i} x_{j} h^{(3)}(I)\left[\bar{e}_{1}, \bar{e}_{i}, \bar{e}_{j}\right] \\
& =\bar{\eta}^{2} x_{1} \sum_{j=2}^{k} x_{j} h^{(3)}(I)\left[\bar{e}_{1}, \bar{e}_{1}, \bar{e}_{j}\right]+\frac{\bar{\eta}^{2}}{2} \sum_{i, j=2}^{k} x_{i} x_{j} h^{(3)}(I)\left[\bar{e}_{1}, \bar{e}_{i}, \bar{e}_{j}\right] .
\end{aligned}
$$

Thus, by Cauchy-Schwarz inequality, (B), (4), (32) and (33),

$$
\begin{aligned}
\left|\bar{h}^{\prime}(I+\bar{\eta} u) \cdot \bar{e}_{1}-\bar{h}^{\prime}\left(I+\bar{\eta} x_{1} \bar{e}_{1}\right) \cdot \bar{e}_{1}\right| & \leq \sqrt{k-1} \bar{\eta}^{3} v M+\frac{k-1}{2} \bar{\eta}^{4} v^{2} M \\
& \leq \xi^{2} \cdot \xi_{k}\left(\sqrt{k-1} \frac{k}{\gamma^{2}}+\frac{k-1}{2} \xi_{k} \frac{k^{2}}{\gamma^{2}}\right) \beta \\
& \leq \xi^{2}\left(\xi_{k} 2 \frac{k^{2}}{\gamma^{2}}\right) \\
& \leq \xi^{2} \theta \beta=\frac{\kappa}{4} \xi^{2} .
\end{aligned}
$$


Next, by (36), (35), (28) and (33), we find:

$$
\begin{aligned}
& \left|\bar{h}^{\prime}\left(I+\bar{\eta} x_{1} \bar{e}_{1}\right) \cdot \bar{e}_{1}-\bar{h}^{\prime}\left(I+\bar{\eta} \sigma \bar{e}_{1}\right) \cdot \bar{e}_{1}\right| \\
& \quad=\left|\bar{\eta} \lambda_{1} \sigma\left(1-\left|x_{1}\right|\right)+\frac{\bar{\eta}^{2}}{2}\left(x_{1}^{2}-1\right) h^{(3)}(I)\left[\bar{e}_{1}\right]^{3}\right| \\
& \quad \leq \bar{\eta}^{3} v^{2}\left(M+\frac{\xi_{k}}{2} M\right) \\
& \quad \leq \xi^{2} \cdot \xi_{k} \frac{k^{2}}{\gamma^{2}}\left(\frac{\beta}{\gamma}+\frac{\xi_{k}}{2} \frac{\beta}{\gamma}\right) \\
& \quad \leq \xi^{2} \theta \beta=\frac{\kappa}{4} \xi^{2} .
\end{aligned}
$$

Thus, for $\xi \leq \xi_{k}$, by (38) and (39), one gets

$$
\begin{aligned}
\left\|P_{V^{k}} \bar{h}^{\prime}(I+\bar{\eta} u)\right\| & \geq\left|\bar{h}^{\prime}(I+\bar{\eta} u) \cdot \bar{e}_{1}\right| \\
& \geq\left|\bar{h}^{\prime}\left(I+\sigma \bar{\eta} \bar{e}_{1}\right) \cdot \bar{e}_{1}\right|-\left|\bar{h}^{\prime}\left(I+\bar{\eta} x_{1} \bar{e}_{1}\right) \cdot \bar{e}_{1}-\bar{h}^{\prime}\left(I+\bar{\eta} \sigma \bar{e}_{1}\right) \cdot \bar{e}_{1}\right| \\
& \quad-\left|\bar{h}^{\prime}(I+\bar{\eta} u) \cdot \bar{e}_{1}-\bar{h}^{\prime}\left(I+\bar{\eta} x_{1} \bar{e}_{1}\right) \cdot \bar{e}_{1}\right| \\
& \geq \frac{\kappa}{2} \xi^{2} .
\end{aligned}
$$

proving claim (31) also in case (B).

Finally, from (19), (40) and the definition of $\xi_{k}$, Theorem follows.

\section{References}

1. Benettin, G., Fassò, F., Guzzo, M.: Nekhoroshev stability of L4 and L5 in the spatial restricted three body problem. Regul. Chaotic Dyn. 3, 56-72 (1998)

2. Benettin, G., Galgani, L., Giorgilli, A.: A proof of Nekhoroshev's theorem for the stability times in nearly integrable Hamiltonian systems. Cel. Mech. 37, 1-25 (1985)

3. Benettin, G., Gallavotti, G.: Stability of motions near resonances in quasi-integrable Hamiltonian systems. J. Stat. Phys. 44, 293-338 (1985)

4. Bounemoura, A., Marco, J.P.: Improved exponential stability for near-integrable quasi-convex Hamiltonians. Nonlinearity 24(1), 97-112 (2011)

5. Gallavotti, G.: Quasi-integrable mechanical systems. In: Osterwalder, K., Stora, R. (eds.) Critical Phenomena, Random Systems, Gauge Theories. Les Houches, Session XLIII, 1984. North-Holland, Amsterdam (1986)

6. Guzzo, M., Chierchia, L., Benettin, G.: The steep Nekhoroshev's theorem and optimal stability exponents (an announcement). Atti Accad. Naz. Lincei Cl. Sci. Fis. Mat. Natur. 25, 293-299 (2014)

7. Guzzo, M., Chierchia, L., Benettin, G.: The steep Nekhoroshev's theorem. Commun. Math. Phys. 342, 569-601 (2016)

8. Guzzo, M., Lega, E., Froeschlé, C.: First numerical investigation of a conjecture by N.N. Nekhoroshev about stability in quasi-integrable systems. Chaos 21(3), 1-13 (2011). paper 033101

9. Guzzo, M.: The Nekhoroshev theorem and long-term stabilities in the solar system. Serb. Astron. J. 190, $1-10$ (2015)

10. Lhotka, Ch., Efthymiopoulos, C., Dvorak, R.: Nekhoroshev stability at $L_{4}$ or $L_{5}$ in the elliptic-restricted three-body problem — application to Trojan asteroid. Mon. Not. R. Astron. Soc. 384(3), 1165-1177 (2008)

11. Lochak, P.: Canonical perturbation theory via simultaneous approximations. Russ. Math. Surv. 47, 57-133 (1992)

12. Lochak, P., Neishtadt, A.: Estimates in the theorem of N.N. Nekhoroshev for systems with quasi-convex Hamiltonian. Chaos 2, 495-499 (1992)

13. Morbidelli, A., Guzzo, M.: The Nekhoroshev theorem and the asteroid belt dynamical system. Celest. Mech. Dyn. Astron. 65, 107-136 (1997)

14. Nekhoroshev, N.N.: Behavior of Hamiltonian systems close to integrability. Funct. Anal. Appl. 5, 338-339 (1971) 
15. Nekhoroshev, N.N.: Behavior of Hamiltonian systems close to integrability. Funk. An. Ego Prilozheniya 5, 82-83 (1971)

16. Nekhoroshev, N.N.: Stable lower estimates for smooth mappings and for gradients of smooth functions. Math USSR Sbornik 19, 425-467 (1973)

17. Nekhoroshev, N.N.: An exponential estimate of the time of stability of nearly-integrable Hamiltonian systems I. Uspekhi Mat. Nauk 32, 5-66 (1977)

18. Nekhoroshev, N.N.: An exponential estimate of the time of stability of nearly-integrable Hamiltonian systems I. Russ. Math. Surv. 32, 1-65 (1977)

19. Nekhoroshev, N.N.: An exponential estimate of the time of stability of nearly-integrable Hamiltonian systems II. Tr. Semin. Petrovsk. 5, 5-50 (1979)

20. Nekhoroshev N.N.: In: Oleinik, O.A. (ed.) Topics in Modern Mathematics, Petrovskii Seminar, No. 5. Consultant Bureau, New York (1985)

21. Niederman, L.: Hamiltonian stability and subanalytic geometry. Ann. Inst. Fourier (Grenoble) 56(3), 795-813 (2006)

22. Pavlovic, R., Guzzo, M.: Fulfillment of the conditions for the application of the Nekhoroshev theorem to the Koronis and Veritas asteroid families. Mon. Not. R. Astron. Soc. 384, 1575-1582 (2008)

23. Pinzari, G.: Aspects of the planetary Birkhoff normal form. Regul. Chaotic Dyn. 18(6), 860-906 (2013)

24. Pöschel, J.: Nekhoroshev estimates for quasi-convex hamiltonian systems. Math. Z. 213, 187 (1993)

25. Sansottera, M., Locatelli, U., Giorgilli, A.: On the stability of the secular evolution of the planar SunJupiter-Saturn-Uranus system. Math. Comput. Simul. 88, 1-14 (2013)

26. Schirinzi, G., Guzzo, M.: On the formulation of new explicit conditions for steepness from a former result of N.N. Nekhoroshev. J. Math. Phys. 54(072702), 1-22 (2013)

27. Schirinzi, G., Guzzo, M.: Numerical verification of the steepness of three and four degrees of freedom hamiltonian systems. Regul. Chaotic Dyn. 20(1), 1-18 (2015)

28. Todorović, N., Guzzo, M., Lega, E., Froeschlé, Cl: A numerical study of the stabilization effect of steepness. Celest. Mech. Dyn. Astr. 110, 389-398 (2011)

29. Zhang, Ke: Speed of Arnold diffusion for analytic Hamiltonian systems. Invent. Math. 186(2), 255-290 (2011)

Publisher's Note Springer Nature remains neutral with regard to jurisdictional claims in published maps and institutional affiliations. 